\title{
Sulle equazioni differenziali del tetraedro dell'ottaedro e dell'icosaedro.
}

\author{
(Memoria di F. BrIoschr, in Milano.)
}

I.

1. Le tre espressioni:

$$
\begin{array}{rlrl} 
& .^{\mathrm{a}} & t & =1+a z^{2} \\
2 .^{\mathrm{a}} & t=\frac{a z^{6}+b}{z^{2}} \\
3 .^{\mathrm{a}} & t=\frac{a z^{12}+b z^{6}+c}{z^{2}}
\end{array}
$$

soddisfano alla equazione differenziale:

$$
t^{3}-1=-\left(\frac{d t}{d z}\right)^{2} R(z)
$$

essendo $R(z)$ una funzione intiera di $z$, purchè per la $2 .^{2}$ sia:

e per la $3 .^{\mathrm{a}}$ sussistano le:

$$
a b^{2}=\frac{4}{27}
$$

$$
b^{2}=20 a c, \quad b c^{2}=2 \frac{5^{3}}{\overline{12}^{3}} .
$$

Il valore della funzione $R$ è nei tre casi :

$$
\begin{array}{rlrl} 
& .^{\mathrm{a}} & R & =\frac{1}{4 a}\left(a^{2} z^{4}+3 a z^{2}+3\right) \\
2 .^{\mathrm{a}} & R & =\frac{1}{16}\left(a z^{6}+4 b\right) \\
3 .^{\mathrm{a}} & R & =\frac{1}{4 \cdot 5^{2}}\left(a z^{18}+\frac{11}{5} b z^{6}+5^{2} c\right) .
\end{array}
$$


Dalla equazione (1) si ha:

$$
\left(t^{3}-1\right)\left(\frac{d z}{d t}\right)^{2}=R(z)
$$

la quale differenziata due volte rispetto a $t$ conduce alla:

$$
2\left(t^{3}-1\right) \frac{d^{3} z}{d t^{3}}+9 t^{2} \frac{d^{2} z}{d t^{2}}+6 t \frac{d z}{d t}=R^{\prime \prime}(z) \frac{d z}{d t} .
$$

Ma dai tre valori superiori di $R(z)$ si ha tosto che indicando con $p$ un coefficiente numerico ha luogo nei tre casi la relazione:

$$
R^{\prime \prime}(z)=\rho\left[t+\frac{1}{2} z \frac{d t}{d z}\right]
$$

essendo in ciascuno di essi:

$$
\rho=\frac{3}{2}, \quad \rho=\frac{5}{8}, \quad \rho=\frac{11}{50} \text { ossia } \rho=\frac{2(n-1)}{(n-2)^{2}} \text { per } n=4,6,12 .
$$

Sostituendo questa espressione di $R^{\prime \prime}(z)$ nella (2) si ottiene la equazione differenziale lineare del terzo ordine seguente:

$$
2\left(t^{3}-1\right) \frac{d^{3} z}{d t^{3}}+9 t^{2} \frac{d^{2} z}{d t^{2}}+(6-\rho) t \frac{d z}{d t}-\frac{1}{2} \rho z=0
$$

Ora posto:

$$
P=\frac{3}{2} \frac{t^{2}}{t^{3}-1}, \quad Q=-\frac{1}{8} \rho \frac{t}{t^{3}-1}
$$

la equazione stessa divisa per $2\left(t^{3}-1\right)$ può scriversi:

$$
\frac{d^{3} z}{d t^{3}}+3 P \frac{d^{2} z}{d t^{2}}+\left(\frac{d P}{d t}+2 P^{2}+4 Q\right) \frac{d z}{d t}+2\left(\frac{d Q}{d t}+2 P Q\right) z=0
$$

la $z$ cioè è eguale ad una forma quadratica (a coefficienti costanti) di due integrali particolari $v_{1}, v_{2}$ della equazione differenziale lineare del secondo ordine:

$$
\frac{a^{2} v}{d t^{2}}+P \frac{d v}{d t}+Q v=0
$$

2. ${ }^{\circ}$ Pel valore di $P$ risultando:

$$
e^{\int P d t}=\sqrt{t^{3}-1}
$$

Annali di Matematica, tomo X. 
se supponiamo $z=v_{1} v_{2}$ ed indichiamo con $Z(t)$ l'integrale:

$$
Z(t)=\int \frac{d t}{z \sqrt{t^{3}-1}}
$$

si hanno per gli integrali particolari $v_{1}, v_{2} \mathrm{i}$ valori:

$$
v_{1}=\sqrt{z} \cdot e^{\frac{1}{2} C Z(t)}, \quad v_{2}=\sqrt{z} \cdot e^{-\frac{1}{2} C Z(t)}
$$

essendo $C$ una costante. L'integrale $Z$ trasformasi per la relazione (1) nel seguente:

$$
Z=\int \frac{d z}{z \sqrt{R(z)}}
$$

cioè le $v_{1}, v_{2}$ sono evidentemente, nei tre casi, funzioni algebriche di $z$.

La equazione differenziale (5) trasformata assumendo come variabile la $z$ dà:

$$
\frac{d^{2} v}{d \tilde{z}^{2}}+\frac{1}{2} \frac{R^{\prime}(z)}{R(z)} \frac{d v}{d z}-\frac{1}{8} \rho \frac{t}{R(z)} v=0
$$

nella quale $t, \rho$ hanno $\mathrm{i}$ valori corrispondenti sopra indicati. Ora dalla $z=v_{1} v_{2}$ e dai valori di $v_{1}, v_{2}$ in funzione di $z$ si hanno le:

$$
\begin{aligned}
1 & =v_{2} \frac{d v_{1}}{d z}+v_{1} \frac{d v_{2}}{d z} \\
\frac{C}{\sqrt{\kappa(z)}} & =v_{2} \frac{d v_{1}}{d z}-v_{1} \frac{d v_{2}}{d z}
\end{aligned}
$$

dalle quali si ottiene la:

$$
2 \frac{d v_{1}}{d z} \frac{d v_{2}}{d z}=\frac{1}{2 z R}\left(R-C^{2}\right)
$$

Ma differenziando di nuovo la prima delle superiori e sostituendo alle $\frac{d^{2} v_{1}}{d z^{2}}$, $\frac{d^{2} v_{2}}{d z^{2}}$ i valori dati dalla (6) si ha facilmente per quest'ultima che:

$$
C^{2}=R-z R^{\prime}+\frac{1}{2} \rho t z^{2}
$$

equazione la quale differenziata rispetto a $z$ riconduce alla (3).

Il valore della espressione $R-z R^{\prime}$ trovasi essere nei tre casi:

$$
1^{0} \quad R-z R^{\prime}=\frac{3}{4 a}-\frac{1}{2} \rho t z^{2}
$$




$$
\begin{array}{ll}
2 .^{\circ} & R-z R^{\prime}=\frac{9}{16} b-\frac{1}{2} \rho t z^{8} \\
3 .^{\circ} & R-z R^{\prime}=\frac{9}{25} c-\frac{1}{2} \rho t z^{2}
\end{array}
$$

nelle quali $\rho$ ha $\mathrm{i}$ valori corrispondenti a ciascuno di essi. I valori di $C$ saranno quindi:

$$
C=\frac{\sqrt{3}}{2 \sqrt{a}}, \quad C=\frac{3}{4} \sqrt{b}, \quad C=\frac{3}{5} \sqrt{c} .
$$

3. ${ }^{\circ}$ Passiamo ora alla determinazione dei tre valori di $Z$ in funzione di z. Nel primo caso posto:

$$
\omega=\frac{1}{a z^{8}}\left[4 \sqrt{a R}-\left(a z^{2}+2\right) \sqrt{3}\right]
$$

si ottiene:

$$
Z=\frac{\sqrt{a}}{\sqrt{3}} \log \omega
$$

quindi pel primo dei valori di $C$ si avranno le:

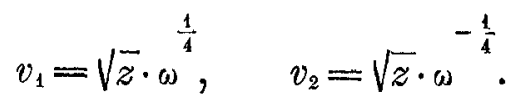

Ma evidentemente :

$$
\omega=\frac{\Phi^{2}(z)}{z^{2}}
$$

essendo:

$$
\varphi(z)=\frac{1}{\sqrt{i a}}\left[\varepsilon \sqrt{1-\varepsilon^{2}(1+a} \overline{\left.z^{2}\right)}+i \varepsilon^{2} \sqrt{1-\varepsilon\left(1+a z^{2}\right)}\right]
$$

posto $i=\sqrt{-1}$ ed indicando con $\varepsilon$ una radice cubica immaginaria della unità. E siccome posto:

$$
\psi(z)=\frac{1}{\sqrt{-i a}}\left[\varepsilon \sqrt{1-\varepsilon^{2}\left(1+a z^{2}\right)}-i \varepsilon^{2} \sqrt{1-\varepsilon\left(1+a z^{2}\right)}\right]
$$

si ha che:

$$
\varphi(z) \psi(z)=z^{2}
$$

sarà :

$$
\frac{1}{\omega}=\frac{\psi^{2}(z)}{z^{2}}
$$

od infine:

$$
v_{1}=\sqrt{\varphi(z)} \quad v_{2}=\sqrt{\psi(z)}
$$


Ponendo nelle funzioni $\varphi, \psi$ in luogo di $1+a z^{2}$ la $t$ si otterranno altresì $\mathrm{i}$ valori di $v_{1}, v_{2}$ in funzione di $t$.

Si noti che pel valore superiore di $\omega$ si ha tosto la:

$$
v_{1}^{4}-v_{2}^{4}=-\frac{2 \sqrt{3}}{a}\left(a z^{2}+2\right)
$$

da cui :

$$
f\left(v_{1}, v_{2}\right)=v_{1}^{4}+2 \sqrt{3} \cdot v_{1}^{2} v_{2}^{2}-v_{2}^{4}=-\frac{4 \sqrt{3}}{a}
$$

e:

$$
h\left(v_{1}, v_{2}\right) \sqrt{3}=v_{1}^{4}-2 \sqrt{3} \cdot v_{1}^{2} v_{2}^{2}-v_{2}^{4}=-\frac{4 \sqrt{3}}{a} t
$$

essendosi posto $t$ in luogo di $1+a z^{2}$.

Nel secondo caso posto:

$$
\omega=\frac{2}{\tilde{z}^{3} \sqrt{a}}[2 \sqrt{R}-\sqrt{b}]
$$

si ottiene:

$$
Z=\frac{2}{3 \sqrt{b}} \log \omega
$$

quindi pel valore di $C$ :

$$
r_{1}=\left(\frac{\%}{z}(z)\right)^{\frac{1}{4}} \quad v_{2}=\left(\frac{\psi(z)}{z}\right)^{\frac{1}{4}}
$$

nelle quali

$$
\zeta(z)=\frac{2}{\sqrt{a}}(2 \sqrt{\bar{l}}-\sqrt{\bar{b}}), \quad \psi(z)=\frac{2}{\sqrt{\bar{a}}}(2 \sqrt{\bar{R}}+\sqrt{\bar{b}}) .
$$

Si osservi che essendo:

$$
\begin{gathered}
\varphi(z) \psi(z)=-z^{6} \quad \varphi(z)-\psi(z)=-4 \sqrt{\frac{b}{a}} \\
\zeta^{2}(z)+\psi^{2}(z)=\frac{2}{a}\left(a z^{6}+8 b\right)
\end{gathered}
$$

si hanno le:

$$
\begin{gathered}
f\left(v_{1}, v_{2}\right)=v_{1} v_{2}\left(v_{1}^{4}-v_{2}^{4}\right)=-4 \sqrt{\frac{b}{a}}=-\frac{8}{\sqrt[4]{108 a^{3}}} \\
6^{2} \cdot h\left(v_{1}, v_{2}\right)=-\left(v_{1}^{8}+14 v_{1}^{4} v_{2}^{4}+v_{2}^{8}\right)=-\frac{16}{a} t
\end{gathered}
$$


posto $t$ in luogo di $\frac{a \varepsilon^{6}+b}{z^{2}}$, in quest' ultima, e rammentando nella prima essere $a b^{2}=\frac{4}{27} \cdot$ Supponendo inoltre

$$
A=1-\sqrt{1-t^{3}}, \quad B=1+\sqrt{1-t^{3}}
$$

i valori di $v_{1}, v_{2}$ espressi in funzione di $t$ sono:

$$
\begin{aligned}
& v_{1}=\frac{1}{\sqrt[8]{3 a}}\left[\sqrt{\varepsilon A^{\frac{1}{3}}+\varepsilon^{2} B^{\frac{1}{3}}}-i \sqrt{\varepsilon^{2} A^{\frac{1}{3}}+\varepsilon B^{\frac{1}{3}}}\right]^{\frac{1}{2}} \\
& v_{2}=\frac{1}{\sqrt[8]{3 a}}\left[\sqrt{\varepsilon A^{\frac{1}{3}}+\varepsilon^{2} B^{\frac{1}{3}}}+i \sqrt{\varepsilon^{2} A^{\frac{1}{3}}+\varepsilon B^{\frac{1}{3}}}\right]^{\frac{1}{2}} .
\end{aligned}
$$

Infine pel terzo caso, posto:

$$
\omega=\frac{5}{2 b *^{6}}\left[4.5^{2} \sqrt{c R}-\frac{11}{5} b z^{6}-2.5^{2} c\right]
$$

si ottiene:

$$
Z=\frac{1}{3 \sqrt{c}} \log \omega
$$

per la quale e pel corrispondente valore di $C$ :

$$
v_{1}=\sqrt{\bar{z} \cdot \omega^{\frac{1}{10}}}, \quad v_{2}=\sqrt{\bar{z} \cdot \omega}{ }^{-\frac{1}{10}}
$$

od indicando con $\varphi(z), \psi(z)$ le funzioni:

$$
\begin{aligned}
& \varphi(z)=\frac{5}{2 b}\left[4.5^{2} \sqrt{c R}-\frac{11}{5} b z^{6}-2.5^{2} c\right] \\
& \psi(z)=\frac{5}{2 b}\left[4.5^{2} \sqrt{c R}+\frac{11}{5} b z^{6}+2.5^{2} c\right]
\end{aligned}
$$

si hanno le:

$$
v_{1}=\left(\frac{\varphi(z)}{z}\right)^{\frac{1}{10}}, \quad v_{2}=\left(\frac{\psi(z)}{z}\right)^{\frac{1}{10}} .
$$

Anche in questo caso essendo:

$$
\begin{gathered}
\varphi(z) \psi(z)=z^{42}, \quad \varphi(z)-\psi(z)=-\frac{1}{b}\left[11 b z^{6}+2.5^{3} c\right] \\
\psi^{2}(z)+\psi^{2}(z)=\frac{4 \cdot 5 \cdot c}{b^{2}}\left[123 a z^{42}+11.5^{2} b z^{6}+5^{5} c\right]
\end{gathered}
$$


si deducono le:

$$
\begin{gathered}
f\left(v_{1}, v_{2}\right)=v_{1} v_{2}\left(v_{1}^{10}+11 v_{1}^{5} v_{2}^{5}-v_{2}^{10}\right)=-2 \cdot 5^{3} \frac{c}{b}=-\frac{5^{3}}{\sqrt[5]{\overline{12}^{3} a^{3}}} \\
\overline{12}^{2} \cdot h\left(v_{1}, v_{2}\right)=-\left[v_{1}^{20}+v_{2}^{20}-228 v_{1}^{5} v_{2}^{5}\left(v_{1}^{10}-v_{2}^{10}\right)+494 v_{1}^{10} v_{2}^{10}\right]=-\frac{5^{5}}{a} t .
\end{gathered}
$$

4. ${ }^{\circ}$ Le tre funzioni $h\left(v_{1}, v_{2}\right)$ trovate sopra sono gli hessiani delle corrispondenti forme binarie $f\left(v_{1}, v_{2}\right)$. Ora importa qui osservare che le tre forme $f\left(v_{1}, v_{2}\right)$ sono costanti e che per ciascuna di esse si hanno le:

$$
\begin{array}{rlrl} 
& .^{\mathrm{a}} & I & =3 \sqrt{3} \frac{h^{3}}{f^{3}} \\
\text { 3. }^{\mathrm{a}} & I & =-3^{3} \cdot 4^{2} \cdot \frac{h^{3}}{f^{4}} \\
& I & =\overline{12}^{3} \frac{h^{3}}{f^{5}}
\end{array}
$$

essendosi posto $I=t^{3}$.

$5^{\circ}$ Se nella terza delle espressioni $t$ del $\S 1 .^{\circ}$ si pone $a=\frac{5^{5}}{12}$ sicchè la corrispondente funzione $f\left(v_{1}, v_{2}\right)$ diventa eguale a -1 , si hanno le

$$
b=\frac{5^{3}}{6}, \quad c=\frac{1}{12}
$$

e ponendo $5 z^{2}=\nu$ si deduce dalla espressione stessa la equazione:

$$
\nu^{6}+10 \nu^{3}-12 t \nu+5=0
$$

vale a dire una equazione Jacobiana del sesto grado per la trasformazione di quinto ordine delle funzioni ellittiche. Se nella equazione stessa supponesi:

$$
I=t^{3}=\frac{4}{27} \frac{\left(1-l^{2}+h^{4}\right)^{3}}{k^{4} k^{\prime}}
$$

si ha:

$$
\nu^{3}=\mu \sqrt{\mu \frac{\lambda \lambda^{\prime}}{\lambda_{k}^{\prime}}}
$$

essendo $\mu$ il moltiplicatore, $\lambda, k$ i moduli. La radice quadrata di una radice qualsivoglia di quella equazione Jacobiana soddisferà quindi la equazione differenzinle lineare del terzo ordine (4). 
II.

1. ${ }^{\circ}$ Posto $I=t^{3}$ la equazione differenziale lineare del secondo ordine della quale ci siamo occupati nel capitolo precedente, si trasforma nella:

$$
\frac{d^{2} v}{d I^{2}}+P \frac{d v}{d I}+Q v=0
$$

posto:

$$
P=\frac{1}{6} \frac{4-7 I}{I(1-I)}, \quad Q=\frac{1}{72} \rho \frac{1}{I(1-I)} .
$$

Siccome è noto, la equazione superiore è una equazione differenziale ipergeometrica, cioè i suoi integrali particolari possono esprimersi per mezzo di serie ipergeometriche.

La forma generale di queste equazioni è la:

$$
\frac{d^{2} y}{d \xi^{2}}+\frac{1-\lambda-(2-\lambda-v) \xi}{\xi\left(1-\xi^{2}\right)} \frac{d y}{d \xi}-\frac{1}{4} \frac{(1-\lambda-\nu)^{2}-\mu^{2}}{\xi(1-\xi)} y=0
$$

e da essa si deduce la (1) supponendo:

$$
y=v, \quad I=\xi, \quad \lambda=\frac{1}{3}, \quad \mu=\frac{\sqrt{2 \rho+1}}{6}, \quad \nu=\frac{1}{2} .
$$

Si ponga ora in quest'ultima:

$$
\xi=\frac{c-b}{c-a} \frac{x-a}{x-b}
$$

la equazione stessa si trasformerà nella:

$$
y^{\prime \prime}+p y^{\prime}+q y=0
$$

dove $y^{\prime}=\frac{d y}{d x}, y^{\prime \prime}=\frac{d^{2} y}{d x^{2}}$, e :

$$
\left.\begin{array}{c}
p=\frac{1-\lambda}{x-a}+\frac{\lambda+v}{x-b}+\frac{1-v}{x-c} \\
q=\frac{(1-\lambda-v)^{2}-\mu^{2}}{4 \Delta}\left[\frac{\Delta}{(x-b)^{2}}+\frac{(b-c)^{2}}{x-a}+\frac{(2 b-c-a)(c-a)}{x-b}-\frac{(b-a)^{2}}{x-c}\right]
\end{array}\right\}
$$

essendo $\Delta=(b-c)(c-a)(a-b)$.

$\mathrm{Ci}$ proponiamo in questo capitolo di determinare $\mathrm{i}$ valori delle $\lambda, \mu, \nu ; a$, $b, c$ pei quali le due equazioni differenziali (1) (3) si trasformano l'una nel- 
l'altra, ossia pei quali un integrale particolare $y$ della seconda si possa dedurre dal corrispondente integrale particolare $v$ della prima per mezzo della relazione:

$$
y=w v
$$

essendo $w$ una funzione di $x$; colla condizione che la $I$ sia una funzione razionale di $x$.

2..$^{\circ}$ Derivando la equazione $y=w v$ due volte rispetto ad $x$ e sostituendo i valori di $y, y^{\prime}, y^{\prime \prime}$ nella (3) si ottiene una equazione differenziale la quale dovendo coincidere colla (1) conduce allo due relazioni:

$$
\begin{aligned}
& P I^{\prime_{2}}=I^{\prime \prime}+\left(2 \frac{w^{\prime}}{w}+p\right) I^{\prime} \\
& Q I^{\prime 2}=\frac{w v^{\prime \prime}}{w}+p \frac{w v^{\prime}}{w}+q .
\end{aligned}
$$

La prima di queste dà tosto:

$$
e^{-\int P d I} \cdot T^{\prime}=\frac{D}{O} \frac{e^{-\int p d x}}{u u^{2}}
$$

le costanti $D, C$ essendo quelle delle due relazioni:

$$
y_{2} y_{1}^{\prime}-y_{1} y_{2}^{\prime}=D e^{-\int p d x}, \quad v_{2} \frac{d v_{1}}{d I}-v_{1} \frac{d v_{2}}{d I}=C e^{-\int P d I} .
$$

Pel valore di $P$ si avrà quindi:

$$
\frac{I^{\prime}}{I^{\frac{2}{3}} \sqrt{1-I}}=\frac{D}{C} \frac{e^{-\int x d l s}}{u^{2}}=\frac{D}{C} \frac{1}{r^{2}}
$$

posto:

$$
w=n e^{-\frac{1}{2} \int t d x}
$$

La seconda relazione per questo valore di $w$ diventa:

$$
Q I^{\prime 2}=\frac{d^{2} \log n}{d x^{2}}+\left(\frac{d \log r_{1}}{d x}\right)^{2}+\frac{1}{2} \Pi
$$

essendo:

$$
\Pi=2 q-\frac{1}{2} p^{2}-p
$$


e se in essa si pone per $Q$ il suo valore e per $I^{\prime}$ il valore dato dalla precedente, si giunge alla:

$$
I^{\frac{1}{3}}=\frac{72 C^{z}}{\rho D^{2}} n^{4}\left[\frac{d^{2} \ln g n}{d x^{2}}+\left(\frac{d \log x}{d x}\right)^{2}+\frac{1}{2} \Pi\right] .
$$

Questa espressione per $I$ dimostra che la condizione posta dover essere $I$ una funzione razionale di $x$ esige sia $x_{1}^{12}$ una funzione razionale; percio ponendo:

$$
x^{12}=\psi(x)
$$

sarà $\psi(x)$ una funzione razionale di $x$, e si avrà:

$$
I=\delta \psi(x) \Phi^{3}(x)
$$

nella quale:

$$
\delta=\left(\frac{C^{2}}{2 \rho D^{2}}\right)^{3}, \quad \Phi(x)=12 \frac{d^{2} \log \psi}{d x^{2}}+\left(\frac{d \log \psi}{d x}\right)^{2}+72 \Pi .
$$

D'altra parte dalla equazione (5) si deduce la:

$$
\frac{I^{\prime}}{\sqrt{I(1-I)}}=\frac{D}{C} \frac{I^{\frac{1}{6}}}{7_{i}^{2}}=\frac{1}{\sqrt{2 \rho}} \sqrt{\Phi}
$$

se quindi da quest'ultima e dalla (6) si elimina la $I$ si otterrà fra le $\psi, p, q$ la relazione:

$$
2 \rho \delta\left[3 \psi \Phi^{\prime}+\psi^{\prime} \Phi\right]^{2}=\psi\left(1-\delta^{\prime} \psi \Phi^{3}\right) .
$$

Le espressioni (4) di $p, q$ danno pel valore di II la:

$$
\Pi=\frac{1}{2} \frac{M(x)}{\psi^{2}(x)}
$$

nella quale:

$$
\varphi(x)=(x-a)(x-b)(x-c)
$$

ed :

$$
M(x)=\Sigma\left(1-\lambda^{2}\right)(a-b)(a-c)(x-b)(x-c)
$$

il segno sommatorio indicando la somma di tre termini formati analogamente al primo. Ora se nella (8) poniamo $\xi$ in luogo di $I$ ritenendo per $\xi$ il valore (2) si ottiene:

$$
\Phi=-2 \rho \frac{(b-c)(h-a)(x-c)(x-a)}{\varphi^{2}}
$$


e quindi:

$$
\Phi-72 \Pi=\frac{1}{\zeta}\left[\frac{32(a-b)(a-c)}{x-a}+\frac{35(b-c)(b-a)}{x-b}+\frac{27(c-a)(c-b)}{x-c}\right]
$$

allorquando nel valore di $\Pi$ si sostituiscono a $\lambda, \mu, \nu$ i valori corrispondenti ad $I=\zeta$. Quest' ultima equazione dimostra dover essere:

$$
\psi(x)=(x-a)^{x}(x-b)^{3}(x-c)^{y}
$$

posto $\alpha=4, \beta=5, \gamma=3$.

Assumendo in generale questa espressione per $\psi(x)$ colla condizione che le a, $\beta, 7$ sieno numeri interi, positivi, si avrà:

$$
12 \frac{d^{2} \operatorname{lng} \dot{y}}{a x^{2}}+\left(\frac{d \log \dot{y}}{d x}\right)^{2}=\frac{L(x)}{\iota^{2}(x)}
$$

essendo:

$$
L(x)=2 \varphi \Sigma\left(\jmath \%(x-a)-\Sigma \alpha(12-a)(x-b)^{2}(x-c)^{2} ;\right.
$$

inoltre indicando con $\Psi(x)$ ed $N(x)$ le due espressioni:

$$
\begin{gathered}
\Psi^{\circ}(x)=L(x)+36 M(x) \\
N(x)=\frac{t^{c}}{y}=(x-a)^{s-\sigma}(x-b)^{\sigma-\beta}(x-c)^{\sigma-\gamma}
\end{gathered}
$$

si arranno per le (6) (9):

$$
\begin{gathered}
I=\partial \frac{\Psi 3(x)}{N(x)} \\
2 \beta \partial\left[3 \% \Psi^{\prime}-\Psi \Sigma(6-\alpha)(x-b)(x-c)\right]^{2}=N-\partial^{\prime} \psi^{3}
\end{gathered}
$$

nella seconda delle quali supponendo ciascuna delle $\alpha, \beta, \gamma$ non maggiori del numero 6, l'uno e l'altro membro sono funzioni inticre di $x$.

Si avrà infine:

$$
w=(x-a)^{\alpha_{1}}(x-b)^{\hat{r}_{1}}(x-c)^{y_{1}}
$$

posto:

$$
\alpha_{1}=\frac{1}{12}[\alpha-6(1-\lambda)], \quad \beta_{1}=\frac{1}{12}[\beta-6(\lambda+\nu)], \quad \gamma_{1}=\frac{1}{12}[\gamma-6(1-\nu)] .
$$

Nelle ipotesi sopra espresse le equazioni (10), (12) risolvono adunque il problema clue abbiamo di mira, mentre la equazione (11) considerata siccome equazione identica stabilisce le necessarie condizioni alle quali devono soddisfare le $\alpha, \beta, \gamma ; \lambda, \mu, \nu ; \alpha, b, c$. 
3. La espressione $\Psi(x)$ è in generale un polinomio del $4^{\circ}$ grado, perciò il primo membro della equazione (11) sarà del $12^{\circ}$ grado, come pure sarà del $12^{\prime \prime}$ grado il secondo termine del secondo membro. Il primo termine del medesimo non potendo essere di grado superiore a 12 dovrà quindi verificarsi la:

$$
g=\alpha+\beta+\gamma \text { non }<6 \text {. }
$$

Supponiamo $g>6$; eguagliando i coefficienti di $x^{12}$ nei due membri della equazione (11) si ha:

$$
2 e g^{2}(g-6)^{2}(g-12)^{2}=-g^{3}(g-12)^{3}
$$

la quale rammentando essere:

$$
\rho=\frac{2(n-1)}{(n-2)^{2}} \quad \text { per } \quad n=4,6,12
$$

è soddisfatta nei due casi:

$$
g=12, \quad g=12 \frac{n-1}{n}
$$

e siccome supponendo $g=6$, dal confronto di quei coefficienti si otterrebbe per $\delta$ un valore numerico costante, il che evidentemente non può sussistere, possiamo concludere che i soli valori di $g$ a considerarsi sono i due superiori (13).

$4 .^{\circ}$ Se nel valore di $\Psi(x)$ si sostituiscono in luogo di $x$ le $a, b, c$ si ottengono le:

$$
\begin{aligned}
& \Psi(a)=\left[36\left(1-\lambda^{2}\right)-\alpha(12-\alpha)\right](a-b)^{2}(a-c)^{2} \\
& \Psi(b)=\left[36\left(1-\mu^{2}\right)-\beta(12-\beta)\right](b-c)^{2}(b-a)^{2} \\
& \Psi(c)=\left[36\left(1-\nu^{2}\right)-\gamma(12-\gamma)\right](c-a)^{2}(c-b)^{2}
\end{aligned}
$$

e siccome operando la stessa sostituzione nella equazione di condizione (11) si hanno:

$$
\mathrm{Y}^{\prime}(a)=0 \quad \text { oppure } \quad \Psi(a)+2 \rho(6-\alpha)^{2}(a-b)^{2}(a-c)^{2}=0
$$

e le analoghe; dovranno sussistere fra le $\lambda, \mu, \nu ; \alpha, \beta, \gamma$ le seguenti relazioni:

$$
\left.\begin{array}{lcll}
\lambda=\frac{6-x}{6} & \text { oppure } & \lambda=\frac{n}{n-2} \frac{6-\alpha}{6} \\
\mu=\frac{6-\beta}{6} & n & \mu=\frac{n}{n-2} \frac{6-\beta}{6} \\
\nu=\frac{6-\gamma}{6} & n & \nu=\frac{n}{n-2} \frac{6-\gamma}{6}
\end{array}\right\}
$$


per $n=4,6,12$. Da queste relazioni, considerando che per la natura del problema le $\lambda, \mu, \nu$ devono essere frazioni numeriche minori dell'unita, si deduce tosto che le $\alpha, \beta, \gamma$ non possono avere valori maggiori di cinque o minori di due.

$5 .^{\circ}$ Consideriamo dapprima il caso in cui:

$$
g=\alpha+\beta+\gamma=12 \text {. }
$$

Il polinomio $\Psi(x)$ si riduce al secondo grado, risultando in questo caso:

$$
L(x)=-\beta \gamma(b-c)^{2}(x-a)^{2}-\gamma \alpha(c-a)^{2}(x-b)^{2}-\alpha \beta(a-b)^{2}(x-c)^{2}
$$

e ciascun membro della (11) diventa del sesto grado.

Si noti che le $\alpha, \beta, \%$ possono permutarsi nei valori di $I, w$, senza alterare i valori stessi, purchè si faccia subire la stessa permutazione alle $a, b, c$; le condizioni trovate sopra per $a, \beta, \%$ non possono perciò dar luogo in questo caso che alle tre combinazioni:

$$
\left.\begin{array}{lll}
\alpha=4, & \beta=5, & \gamma=3 \\
\alpha=4, & \beta=4, & \gamma=4 \\
\alpha=5, & \beta=5, & \gamma=2 .
\end{array}\right\}
$$

La espressione $\Psi(x)$ essendo del secondo grado, non possono essere insieme $\Psi(a)=0, \Psi(b)=0, \Psi(c)=0$. Supponiamo dapprima sieno nulli due fra quei valori, per esempio:

$$
\Psi(a)=0, \quad \Psi(c)=0
$$

si avranno le:

$$
\lambda=\frac{6-x}{6}, \quad \mu=\frac{n}{n-2} \frac{6-\beta}{6}, \quad \nu=\frac{6-\gamma}{6}
$$

per le quali il valore di $\Psi(x)$ diventa:

$$
\Psi(x)=-2 p(6-\xi)^{2}(b-c)(b-a)(x-c)(x-a) .
$$

I valori di $I, w$ sono in questo caso:

$$
I=-8 \partial \rho^{3}(6-\beta)^{6}(b-c)^{3}(b-a)^{3} \frac{(x-c)^{3}(x-a)^{3}}{N}, \quad w=1
$$

e la equazione di condizione (11) si riduce alla:

$8 \delta \rho^{3}(6-\beta)^{4} \Delta(b-a)(b-c)\left[(\alpha-3)^{2}(a-b)(x-c)+(y-3)^{2}(b-c)(x-a)\right]=\frac{N}{(x-a)(x-c) p}$

essendo come precedentemente $\Delta=(b-c)(c-a)(a-b)$. 
La $1 .^{a}$ combinazione $\alpha=4, \beta=5, \gamma=3$ conduce alla:

$$
I=\frac{c-b}{c-a} \frac{x-a}{x-b}
$$

ossia alla $I=\xi$, come doveva essere per quanto si è veduto nel $\S 2 .^{\circ}$; e la combinazione $\alpha=4, \beta=4, \gamma=4$ dà per $I$ il valore:

$$
I=-4 \frac{(b-c)(b-a)}{(a-c)^{2}} \cdot \frac{(x-c)(x-a)}{(x-b)^{2}} .
$$

La terza combinazione (15) non può sussistere nel caso attuale, come provasi sostituendo per $\alpha, \beta, \gamma$ questi valori nella (17).

Se suppongonsi invece:

$$
\Psi(a)=0 \quad \Psi(b)=0
$$

vale a dire si permutano le $b, c ; \beta, \gamma ; \mu, \nu ;$ sono:

$$
\lambda=\frac{6-x}{6}, \quad \mu=\frac{6-\beta}{6}, \quad \nu=\frac{n}{n-2} \frac{6-\gamma}{6}
$$

e si ha per la (12):

$$
w=\left(\frac{x-c}{x-6}\right)^{\frac{6-y}{0(n-2)}}
$$

e permutando le $\beta, \gamma ; b, c$ nel valore superiore (16) di $\Psi$ si otterrà:

$$
\Psi(x)=-2 \rho(6-\gamma)^{2}(c-b)(c-a)(x-b)(x-a)
$$

ed analogamente per la (17). Si giungerà così ai due valori di $I$ corrispondenti alle prime due combinazioni (15), nelle quali si permutino le $\beta, \gamma$; ciò̀

$$
I=\frac{b-c}{b-a} \frac{x-a}{x-c}, \quad I=-4 \frac{(c-b)(c-a)}{(a-b)^{2}} \cdot \frac{(x-b)(x-a)}{(x-c)^{2}}
$$

non potendo sussistere anche nel caso attuale la terza combinazione (15).

Riassumendo si ha che supponendo eguali a zero due fra $\mathrm{i}$ valori di $\Psi(x)$ corrispondenti ad $x=a, x=b, x=c$ si presentano i seguenti risultati:

si hanno:

$$
\begin{aligned}
& \text { per } \alpha=4, \quad \beta=5, \quad \gamma=3 \\
& \lambda=\frac{1}{3}, \quad \mu=\frac{n}{6(n-2)}, \quad \nu=\frac{1}{2}
\end{aligned}
$$

$$
y=v, \quad I=\frac{c-b}{c-a} \frac{x-a}{x-b}=\xi
$$


e

$$
\operatorname{per} \begin{cases}\alpha=4, & \beta=4, \quad \gamma=4 \\ \lambda=\frac{1}{3}, & \mu=\frac{1}{3},\end{cases}
$$

sono:

$$
y=\left(\frac{x-c}{x-b}\right)^{\frac{1}{3(n-2)}} v, \quad I=-4 \frac{(c-b)(c-a)}{(a-b)^{2}} \frac{(x-b)(x-a)}{(x-c)^{2}}=-\frac{4 b}{(1-\xi)^{2}} .
$$

Negli altri due casi le $\mu, \nu$ si permutano, i valori di $I$ si ottengono dai superiori cambiando la $\xi$ in $\frac{\zeta}{\zeta-1}$ e nell'uno è $y=v$, nell'altro:

$$
y=\left(\frac{x-c}{x-b}\right)^{\frac{1}{6(n-2)}} v
$$

Passiamo a considerare il caso in cui una sola delle funzioni $\Psi(x)$ corrispondenti ad $x=a, b, c$ sia nulla. Supponiamo sia:

si hanno le:

$$
\Psi(c)=0
$$

$$
\lambda=\frac{n}{n-2} \frac{6-\%}{6}, \quad \mu=\frac{n}{n-2} \frac{6-8}{6}, \quad \nu=\frac{6-\gamma}{6}
$$

ed il valore di $\Psi(x)$ :

$$
\Psi(x)=-2 \beta(b-a)(x-c) R(x)
$$

essendo :

$$
R(x)=(6-\beta)^{2}(b-c)(x-a)+(6-a)^{2}(c-a)(x-b) .
$$

Sieno $\alpha=4, \beta=5, \gamma:=3$ la equazione di condizione (11) si riduce alla:

$$
8 \cdot 27 \cdot \delta p^{3} \Delta(b-c)(a-b)^{2}=1
$$

per la quale alle:

$$
\lambda=\frac{n}{3(n-2)}, \quad \mu=\frac{n}{6(n-2)}, \quad \nu=\frac{1}{2}
$$

corrispondono i valori:

$$
\begin{gathered}
I=-\frac{1}{27} \frac{a-b}{(c-a)(c-b)^{3}} \frac{[(b-c)(x-a)+4(c-a)(x-b)]^{3}}{(x-a)^{2}(x-b)}=-\frac{1}{27} \frac{\left(\frac{y}{2}-4\right)^{3}}{\frac{6}{3}^{2}} \\
y=\left(\frac{x-a}{x-b}\right)^{\frac{1}{3(u-2)}} v .
\end{gathered}
$$


In secondo luogo supponendo $\alpha=5, \beta=5, \gamma=2$ si hanno le:

ed i ralori:

$$
\lambda=\frac{n}{6(n-2)}, \quad \mu=\frac{n}{6(n-2)}, \quad \nu=\frac{2}{3}
$$

$$
\begin{gathered}
I=-\frac{1}{4} \frac{(b-a)^{2}}{(c-a)(c-b)} \frac{(x-c)^{2}}{(x-a)(x-b)}=-\frac{1}{4} \frac{\left(1-\frac{\zeta}{2}\right)^{2}}{\xi} \\
y=\left(\frac{x-a}{x-b}\right)^{\frac{1}{b(n-2)}} v .
\end{gathered}
$$

Infine ponendo $\alpha=3, \beta=5, \gamma=4$ e permutando le $a, c$ si ottengono per:

$$
\lambda=\frac{1}{3}, \quad \mu=\frac{n}{6(n-2)}, \quad \nu=\frac{n}{2(n-2)}
$$

le:

$$
\begin{gathered}
I=-\frac{1}{4^{3}} \frac{b-c}{(a-c)(a-b)^{3}} \frac{(x-a)[(b-c)(x-a)+8(a-c)(x-b)]^{3}}{(x-b)(x-c)^{3}}=-\frac{1}{4^{3}} \frac{\zeta(\zeta+8)^{3}}{(1-\xi)^{3}} \\
y=\left(\frac{x-c}{x-b}\right)^{\frac{1}{2(z-2)}} v .
\end{gathered}
$$

Le altre due combinazioni $\alpha=\beta=\gamma=4 ; \alpha=2, \beta=5, \gamma=5$ essendo escluse per la sussistenza della (11) non si hanno a considerare altri casi nella ipotesi cho una sola delle $\Psi(a), \Psi(b), \Psi(c)$ sia nulla.

Supponiamo da ultimo che nessuna di queste funzioni sia eguale a zero; si avranno allora le:

$$
\lambda=\frac{n}{n-2} \frac{6-\mu}{6}, \quad \mu=\frac{n}{n-2} \frac{6-\beta}{6}, \quad \nu=\frac{n}{n-2} \frac{6-Y}{6}
$$

e:

$$
\psi=-2 \beta\left[\Sigma \beta \gamma(b-c)^{2}(x-a)^{2}+36 \Sigma(a-b)(a-c)(x-b)(x-c)\right] .
$$

Se $\alpha=\beta=\gamma=4$ si ottengono cosi le:

ed :

$$
\lambda=\frac{n}{3(n-2)}, \quad \mu=\frac{n}{3(n-2)}, \quad \nu=\frac{n}{3(n-2)}
$$

$$
\begin{gathered}
I=\frac{4}{27} \frac{1}{\Delta^{2}(c-a)^{2}} \frac{\left[(a-b)(a-c)(x-b)(x-c)+(b-c)^{2}(x-a)^{2}\right]^{3}}{(x-a)^{2}(x-b)^{2}(x-c)^{2}}=\frac{4}{27} \frac{\left(1-\xi+\xi^{2}\right)^{3}}{\xi^{2}(1-\zeta)^{2}} \\
y=\left[\frac{(x-a)(x-c)}{(x-b)^{2}}\right]^{\frac{1}{3(n-2)}} v .
\end{gathered}
$$


L'altra combinazione $\alpha=\beta=5, \gamma=2$ dà luogo alle:

$$
\begin{gathered}
\lambda=\mu=\frac{n}{6(n-2)}, \quad \nu=\frac{2 n}{3(n-2)} \\
I=\frac{1}{4 \cdot 27} \cdot \frac{1}{\Delta(b-a)^{3}} \frac{\left[16(c-a)(c-b)(x-a)(x-b)+(a-b)^{2}(x-c)^{2}\right]^{3}}{(x-a)(x-b)(x-c)^{4}}=\frac{1}{4 \cdot 27} \frac{\left(1+14^{2}+\xi 2 \cdot 3\right.}{\xi(1-\xi)^{4}} \\
y=\left[\frac{(x-a)(x-c)^{4}}{(x-b)^{5}}\right]^{\frac{1}{(n-2)}} v .
\end{gathered}
$$

La supposizione $\alpha=4, \beta=5, \gamma=3$ non è sussistente in questo caso come si dimostra tosto colla equazione di condizione (11).

Riassumendo i risultati di questo paragrafo possiamo concludere che allorquando sia:

$$
a+\beta+y=12
$$

1. Se due dei valori di $\Psi(x)$ corrispondenti ad $x=a, b, c$ sono nulli possono essere:

e quindi :

$$
\begin{array}{lll}
\alpha=4, & \beta=5, & \gamma=3 \\
\alpha=4, & \beta=4, & \gamma=4
\end{array}
$$

$$
\begin{array}{lll}
\lambda=\frac{1}{3}, & \mu=\frac{n}{6(n-2)}, & \nu=\frac{1}{2} \\
\lambda=\frac{1}{3}, & \mu=\frac{n}{3(n-2)}, & \nu=\frac{1}{3} .
\end{array}
$$

$2 .^{\circ}$ Se uno solo dei suddetti valori è nullo, possono essere:

$$
\begin{array}{llll}
\alpha=4, & \beta=5, & \gamma=3 & \text { oppure } \quad \alpha=3, \quad \beta=5, \quad \gamma=4 \\
\alpha=5, & \beta=5, & \gamma=2
\end{array}
$$

per le quali:

$$
\lambda=\frac{n}{3(n-2)}, \quad \mu=\frac{n}{6(n-2)}, \quad \nu=\frac{1}{2}
$$

oppure

$$
\begin{array}{lll}
\lambda=\frac{n}{2(n-2)}, & \mu=\frac{n}{6(n-2)}, & \nu=\frac{1}{3} \\
\lambda=\frac{n}{6(n-2)}, & \mu=\frac{n}{6(n-2)}, & \nu=\frac{2}{3} .
\end{array}
$$


3. Infine se nessuno di quei valori è nullo: sussistono le:

$$
\begin{array}{lll}
\alpha=4, & \beta=4, & \gamma=4 \\
\alpha=5, & \beta=5, & \gamma=2
\end{array}
$$

ed in conseguenza:

$$
\begin{array}{lll}
\lambda=\frac{n}{3(n-2)}, & \mu=\frac{n}{3(n-2)}, & \nu=\frac{n}{3(n-2)} \\
\lambda=\frac{n}{6(n-2)}, & \mu=\frac{n}{6(n-2)}, & \nu=\frac{2 n}{3(n-2)} .
\end{array}
$$

Devesi notare che il primo gruppo di valori delle $\lambda, \mu, \nu$ soddisfa alla:

$$
\lambda+\frac{n-2}{n} \mu+\nu=1
$$

il secondo alla:

$$
\frac{n-2}{n} \lambda+\frac{n-2}{n} \mu+\nu=1
$$

ed il terzo alla:

$$
\lambda+\mu+\nu=1 \text {. }
$$

Nelle ipotesi di $n=4,6$ alcuni fra i valori superiori di $\lambda, \mu, 2$ devono escludersi se $i$ valori stessi si sottopongono alla condizione di essere minori dell'unità.

6. Esaurito così il caso di $g=12$ passiamo a considerare l'altro pel quale, come si è dimostrato, si ha:

$$
g=\alpha+\beta+\gamma=12 \frac{n-1}{n} .
$$

Nel medesimo $\Psi(x)$ essendo del quarto grado, potranno essere insieme:

$$
\Psi(a)=0, \quad \Psi(b)=0, \quad \Psi(c)=0
$$

e quindi :

$$
\lambda=\frac{6-\alpha}{6}, \quad \mu=\frac{6-\beta}{6}, \quad \nu=\frac{6-\gamma}{6} .
$$

Il valore di $\Psi(x)$ diventa cos̀̀ :

$$
\Psi(x)==(A x+B) \varphi(x)
$$

essendo:

$$
\begin{gathered}
A=-g(12-g), \quad B=(12-g)[(g-2 \alpha) a+(g-2 \beta) b+(g-2 \gamma) c] \\
\text { Annali di Matematica, tomo X. }
\end{gathered}
$$


e l'equazione di condizione (11) si trasforma nella:

$$
2 \rho \partial[3 A \varphi-(A x+B) \Sigma(3-\alpha)(x-b)(x-c)]^{2}=\frac{N}{\varphi^{2}}-\delta \varphi(A x+B)^{3}
$$

nella quale il grado di $N$ è $\frac{6(n+2)}{n}$, e perciò sempre superiore a 6 . Si può quindi supporre sia per $n=4$ che per $n=6$ oppure $n=12$ che una almeno delle $\alpha, \beta, \%$ per esempio la $\gamma$ sia eguale a 3 ; l'equazione di condizione superiore diventa allora divisibile per $x-c$ e si ha:

$$
\begin{gathered}
2 \rho^{\partial}(x-c)\{3 A(x-a)(x-b)-(A x+B)[(3-a)(x-b)+(3-\beta)(x-a)]\}^{2}= \\
=\frac{N}{(x-c) \xi^{2}}-\partial(x-a)(x-b)(A x+B)^{3} .
\end{gathered}
$$

Sia $n=4$, dovrà essere $\alpha+\beta=6$ quindi $x=\beta=3$ oppure $\alpha=4, \beta=2$. Nel primo caso l'equazione di condizione si riduce alla:

$$
3 \cdot 9^{4} \cdot \partial^{2} g(x)=1+9^{3} \cdot j(3 x-a-b-c)^{3}
$$

la quale è soddisfatta se:

$$
a^{2}+b^{2}+c^{2}=b c+c a+a b
$$

e:

$$
3 \cdot 9^{3} \cdot j\left[a(b-c)^{2}+b(c-a)^{2}+c(a-b)^{2}\right]=1 .
$$

Si avranno così le:

$$
\begin{gathered}
\lambda=\mu=\nu=\frac{1}{2} \\
I=-\frac{1}{3} \frac{(3 x-a-\gamma-c)^{3}}{a(b-c)^{3}+b(c-a)^{2}+c(a-b)^{2}}, \quad y=\frac{v}{(x-b)^{\frac{1}{3}}} .
\end{gathered}
$$

In sccondo luogo supponendo $\alpha=4, \beta=2$; l'equazione di condizione conduce alle:

e percio:

$$
a-4 b+3 c=0, \quad 12 \cdot 9^{4} \cdot \delta(b-c)^{3}=1
$$

$$
\begin{gathered}
x=\frac{1}{3}, \quad \mu=\frac{2}{3}, \quad y=\frac{1}{-2} \\
I=-\frac{1}{4} \frac{(x-a)(x-b)^{2}}{(b-c)^{3}}, \quad y=\frac{v}{(x-b)^{\frac{1}{4}}} .
\end{gathered}
$$

Per $n=6$, essendo $\alpha+\beta=7$ potranno essere $\alpha=4, \beta=3 ; \alpha=5, \beta=2$. Nel 
primo caso la equazione di condizione condurrà ad una relazione fra le $a, b$, $c$ ed alla determinazione del valore di ó; e cioè :

$$
8(a-b)(a-c)+27(b-c)^{2}=0, \quad \delta=\frac{5}{4 \cdot 27 \cdot(2 a-b-c)^{4}}
$$

e si avranno le:

$$
\begin{aligned}
\lambda=\frac{1}{3}, \quad \mu=\nu=\frac{1}{2}, \quad I & =-\frac{5 \cdot 4^{2}}{3^{3}} \frac{(x-a)(5 x-a-2 b-2 c)^{3}}{(2 a-b-c)^{4}} \\
y & =\frac{v}{(x-b)^{\frac{1}{6}}} .
\end{aligned}
$$

Il secondo caso $\alpha=5, \beta=2$ non può sussistere; ma devesi inoltre considerare la combinazione $\alpha=\beta=4, \gamma=2$ per la quale sono:

$$
2 c-a-b=0 ; \quad \lambda=\mu=\frac{1}{3}, \quad \nu=\frac{2}{3}
$$

ed:

$$
I=-4^{3} \cdot \frac{(x-c) \varphi(x)}{(a-b)^{4}}, \quad y=\frac{v}{(x-b)^{\frac{1}{6}}} .
$$

Infine se $n=12$ e quindi $g=11$ si hanno dapprima $\alpha=4, \beta=4, \gamma=3$ e quindi $\lambda=\frac{1}{3}, \mu=\frac{1}{3}, \nu=\frac{1}{2}$. Anche in questo caso si ha una equazione di condizione fra le $a, b, c$, ciò̀ la:

$$
135(c-a)(c-b)+64(a-b)^{2}=0
$$

e saranno:

$$
I=\frac{\overline{11}^{2}}{3^{3} \cdot 4^{3}} \frac{(x-a)(x-b)(11 x-3 a-3 b-5 c)^{3}}{(2 c-x-b)^{5}}, \quad y=\frac{v}{(x-b)^{\frac{1}{19}}} .
$$

Le altre combinazioni devono essere escluse.

Supponiamo in secondo luogo che due fra i valori di $\Psi(x)$ corrispondenti ad $x=a, b, c$ sieno nulli; sieno ciò̀ :

e quindi:

$$
\Psi(a)=0, \quad \Psi(c)=0
$$

$$
\lambda=\frac{6-\alpha}{6}, \quad \mu=\frac{n}{n-2} \frac{6-\beta}{6}, \quad \nu=\frac{6-\gamma}{6} .
$$

Il valore di $\Psi(x)$ sarà:

$$
\Psi(x)=(x-a)(x-c) R(x)
$$


indicando $P(x)$ il polinomio:

$R(x)=(12-g)(x-b)[-g x+(g-2 \alpha) a+(g-2 \beta) b+(g-2 \gamma) c]-2 \rho(6-\beta)^{2}(b-a)(b-c)$

e la equazione di condizione si trasformerà nella:

$$
\begin{gathered}
2 p o\left\{3 c R^{\prime}-R[(3-a)(x-b)(x-c)+(6-\beta)(x-c)(x-a)+(3-y)(x-a)(x-b)]\right\}^{2}= \\
=\frac{N}{(x-a)^{2}(x-c)^{2}}-\delta(x-a)(x-c) R^{3} .
\end{gathered}
$$

Suppongasi ora dapprima $y=2$, posto in quest' ultima $x=c$, vedesi tosto dover essere $R(c)=0$, e quindi:

$$
(12-g)[(g-2 a) a+(g-2 \xi) b-4 c]+2 \beta(6-\beta)^{2}(b-a)=0
$$

relazione lineare fra le $a, b, c$ che deve verificarsi pei tre valori di $n$; ed il valore di $\Psi(x)$ diventa:

$$
\Psi(x)=-\left[g(12-g)(x-b)+2 \rho(6-\xi)^{2}(b-a)\right](x-a)(x-c)^{2} .
$$

Sia $n=12$ quindi $g=11$, sarà $\alpha=4, \beta=5$ da cui:

$$
\lambda=\frac{1}{3}, \quad \mu=\frac{1}{5}, \quad \nu=\frac{2}{3} .
$$

Fra le $a, b, c$ dorrà sussistere la relazione (18) ossia la:

$$
16 a+9 b-25 c=0
$$

ed il valore di $R(x)$ sarà:

$$
R(x)=-\frac{11}{25}\left[\tilde{5}^{2}(x-b)+b-a\right](x-c) .
$$

Posto questo valore nella equaziono di condizione questa risulterà dapprima nuovamente divisibile per $(x-c)^{2}$, ed avendo riguardo alla (19) la equazione stessa si riduce alla:

$$
\frac{\overline{11}^{3} \cdot 3^{6} \cdot 4^{3}}{5^{10}} \circlearrowright(a-b)^{5}=1
$$

per la quale si avrà:

ed:

$$
I=\frac{5)^{4}}{30 \cdot 4^{3}} \frac{(x-a)(x-c)^{2}[25 x-a-24 b]^{3}}{(a-b)^{5}(x-b)}
$$

$$
y=\frac{1}{, \frac{1}{10}} v
$$


Sieno in secondo luogo per $n=12$

$$
\alpha=4, \quad \beta=4, \quad \gamma=3 \quad \text { e quindi } \lambda=\frac{1}{3}, \quad \mu=\frac{2}{5}, \quad \nu=\frac{1}{2}
$$

si ha:

$$
R(x)=(x-b)(-11 x+3 a+3 b+5 c)-\frac{4 \cdot 11}{25}(b-a)(b-c)
$$

- posto questo valore nella equazione di condizione si ottengono da essa le due sole relazioni:

$$
\begin{aligned}
& 64 a-189 b+125 c=0 \\
& \frac{3^{3} \cdot 4^{3} \cdot 7^{7} \cdot \overline{11}^{3}}{5^{15}} \delta^{3}(a-b)^{5}=1
\end{aligned}
$$

per le quali:

$$
I=\frac{1}{3^{3} \cdot 4^{3} \cdot 7^{7}} \frac{\left[5^{3}(x-b)(25 x-a-24 b)-4^{4}(a-b)^{2}\right]^{3}(x-a)}{(b-a)^{5}(x-b)^{2}}
$$

ed $y$ como pel caso precedente.

Suppongasi ora un solo valore di $\Psi^{\prime}(x)$ per $x=a, b, c$ eguale a zero. Sia $\Psi(a)=0$ ed in conseguenza:

$$
\lambda=\frac{6-x}{6}, \quad \mu=\frac{n}{n-2} \frac{6-\beta}{6}, \quad \nu=\frac{n}{n-2} \frac{6-\gamma}{6}
$$

sarà :

essendo:

$$
\Psi(x)=(x-a) R(x)
$$

$$
\begin{aligned}
R(x)= & (12-g)(x-b)(x-c)[-g x+(g-2 \alpha) \alpha+(g-2 \beta) b+(g-2 \gamma) c]- \\
& -2 \rho(b-c)\left[(6-\beta)^{2}(b-a)(x-c)-(6-\gamma)^{2}(c-a)(x-b)\right] .
\end{aligned}
$$

La equazione di condizione (11) diventerà in questo caso:

$$
\begin{gathered}
2 \rho{ }^{\prime}\left\{3 \varphi R^{\prime}-R[(3-a)(x-b)(x-c)+(6-\beta)(x-c)(x-a)+(6-\gamma)(x-a)(x-b)]\right\}^{2} \\
=\frac{N}{(x-a)^{2}}-\delta^{2}(x-a) R^{3} .
\end{gathered}
$$

Ora se poniamo nella medesima:

e quindi :

$$
\alpha=4, \quad \beta=4, \quad \gamma=3
$$

$$
\lambda=\frac{1}{3}, \quad \mu=\frac{2}{5}, \quad \nu=\frac{3}{5}
$$


si ha facilmente che allorquando sieno:

$$
\begin{aligned}
5 a+27 b-32 c & =0 \\
\frac{3^{3} \cdot 4^{7} \cdot \overline{11}^{3}}{5^{5}} \partial(c-b)^{5} & =1
\end{aligned}
$$

la equazione stessa è soddisfatta. Supponendo quindi che fra le $a, b, c$ sussista l'equazione lineare superiore si hanno le:

$$
\begin{gathered}
I=\frac{1}{3^{3} \cdot 4^{7} \cdot 5^{4}} \frac{(x-a)\left[25(5 x+6 b-11 c)(x-b)(x-c)-(b-c)^{2}(115 x-243 b+128 c)\right]^{3}}{(b-c)^{5}(x-b)^{2}(x-c)^{3}} \\
y=\left[\frac{(x-c)^{3}}{(x-b)^{3}}\right]^{\frac{1}{60}} v .
\end{gathered}
$$

Così se :

$$
\theta=4, \quad \xi=5, \quad \gamma=2
$$

dalle quali:

$$
i=\frac{1}{3}, \quad \mu=\frac{1}{5}, \quad \nu=\frac{4}{5}
$$

si ottiene la relazione lineare:

$$
80 a+b-81 c=0
$$

che insieme colla:

$$
3^{12} \cdot 4^{3} \cdot \overline{11} \cdot 3(a-c)^{5}=1
$$

rendono soddisfatta l'equazione di condizione, e si arranno le:

$$
\begin{gathered}
I=\frac{1}{4^{3} \cdot 3^{12}} \frac{(x-a)\left[(x-16 a+15 c)^{3}-5 \cdot 27 \cdot(c-a)(x-c)^{2}\right]^{2}}{(x-b)(x-c)^{4}} \\
y=\left[\frac{(x-c)^{4}}{(x-b)^{3}}\right]^{\frac{1}{60}} v .
\end{gathered}
$$

Infine se:

$$
\alpha=2, \quad \beta=5, \quad \gamma=4
$$

sono:

$$
\lambda=\frac{2}{3}, \quad \mu=\frac{1}{5}, \quad \nu=\frac{2}{5} ;
$$

l'equazione di condizione conduce alle:

$$
\begin{gathered}
25 a+2 b-27 c=0 \\
2 \cdot 3^{3} \cdot 4^{3} \cdot \overline{11}^{3} o^{2}(a-c)^{5}=1
\end{gathered}
$$


per le quali:

$$
\begin{gathered}
I=\frac{1}{2 \cdot 3^{3} \cdot 4^{3}} \frac{(x-a)^{2}\left[(x-c)^{2}-14(c-a)(x-c)+25(c-a)^{2}\right]^{3}}{(c-a)^{5}(x-b)(x-c)^{2}} \\
y=\left[\frac{(x-c)^{2}}{(x-b)^{7}}\right]^{\frac{1}{60}} v .
\end{gathered}
$$

Assumendo per $\xi$ il valore (2) come precedentemente si otterranno così fra le $I$ e

$$
\begin{aligned}
& \text { per } \lambda=\frac{1}{3}, \quad \mu=\frac{1}{5}, \quad \nu=\frac{2}{3} \\
& I=-\frac{27}{4} \frac{\zeta(1-\xi)^{2}(128-3 \xi)^{3}}{(9 \xi+16)^{5}} \\
& \text { per } \quad \lambda=\frac{1}{3}, \quad \mu=\frac{2}{5}, \quad \nu=\frac{1}{2} \\
& I=-\frac{\xi\left[4 \cdot \overline{27} \bar{\zeta}^{2}-7 \cdot 13 \cdot 27 \cdot \xi-7 \cdot 8^{3}\right]^{3}}{(184 \xi-64)^{5}} \\
& \text { per } \lambda=\frac{1}{3}, \quad \mu=\frac{2}{5}, \quad \nu=\frac{3}{5} \\
& I=-\frac{1}{4 \cdot 5^{4}} \frac{\left.\xi_{[27}^{2} \xi^{3}-5^{2} \cdot 27 \cdot \xi_{2}+5^{3} \cdot 23 \cdot \xi-5^{4}\right]^{3}}{\left(1-\xi^{3}\right)^{3}(5+27 \xi)^{5}} \\
& \text { per } \lambda=\frac{1}{3}, \quad \mu=\frac{1}{5}, \quad \nu=\frac{4}{5} \\
& I=-\frac{1}{4 \cdot 5^{5}} \frac{\xi\left[\xi^{3}-6 \cdot 5^{2} \cdot 11 \cdot \xi^{2}+3 \cdot 5^{3} \cdot 71 \cdot \xi+2 \cdot 4^{3} \cdot 5^{4}\right]^{3}}{(1-\xi)^{4}(\xi+80)^{5}}
\end{aligned}
$$

infine

$$
\begin{gathered}
\operatorname{per} \lambda=\frac{2}{3}, \quad \mu=\frac{1}{5}, \quad \nu=\frac{2}{5} \\
I=-\frac{1}{4^{3}} \frac{\xi^{2}\left[{ }^{2}-16 \cdot 17 \cdot \zeta+8 \cdot 5^{3}\right]^{3}}{(1-\xi)^{2}(2 \zeta+25)^{5}} .
\end{gathered}
$$

Notiamo da ultimo che se nessuno dei valori di $\Psi(x)$ corrispondenti ad $x=a$, $b$, c è nullo, non sussistono valori di $\alpha, \beta, \gamma$ e perciò di $\lambda, \mu, \nu$ che soddisfino l'equazione di condizione (11). 


\section{III.}

1. Indicando con $f\left(y_{1}, y_{2}\right)$ la forma binaria che si ottiene sostituendo le $y_{1}, y_{2}$ alle $v_{1}, v_{2}$ nelle forme considerate al capo I, rammentando essere $y=v v$ si ha:

$$
f\left(y_{1}, y_{2}\right)=u^{n} f\left(v_{1}, v_{z}\right)
$$

per $n=4,6,12$; ed essendo $f\left(v_{1}, v_{2}\right)$ nei tre casi eguali ad una costante $G$, sarà :

$$
f\left(y_{1}, y_{2}\right)=G w^{n}
$$

nella quale $w$ ha il valore (12).

Cosi dalla equazione (5) del precedente capitolo, osservando essere $n^{12}=\psi(x)$ si ottiene la:

$$
\frac{\lambda I}{I^{\frac{2}{3}} \sqrt{1-1}}=\frac{D}{C} \frac{d x}{\sqrt[6]{(x-a)^{\alpha}(x-b) y^{2}(x-c)^{2}}}
$$

e quindi per ciascuno dei valori di $\alpha, \beta, \%$ sopra considerati si hanno le corrispondenti relazioni fra $I$ ed $x$ che riducono alle funzioni ellittiche il trascendente del secondo membro.

Infine dai valori di $w$ o dalle relazioni trovate fra $y$ e $v$ si ottengono gli integrali delle varie equazioni differenziali lineari del secondo ordine della forma (3), supposti noti gli integrali particolari $v_{1}, v_{2}$ di cui $i$ valori furono dati nel capitolo $\mathrm{I}$.

Questc equazioni differenziali sono quelle che denominiamo del tetraedro, dell'ottaedro e dell'icosaedro secondo che $n=4,6,12$ per le relazioni scoperte dal prof. Scuwarz fra le medesime e gli indicati corpi regolari nella sua importante Memoria: Ueber diejenigen Fälle, in velchen die Gaussische hypergcometrische Reile eine algebraische Function ihres vierten Elementes darstellt (Journal für die Mathematik, Bd. 75). Si possono altresì consultare intormo all'argomento le interessanti ricerche del prof. KLEIN pubblicate nei Mathematische Annalen, Bd. 11, 12; ed una mia Nota nello stesso periodico inserta nel rolume XI.

Scttembre, 1880 . 